\title{
Non-Gromov hyperbolicity of asymptotic Teichmuller spaces
}

\author{
Jinhua Fan \\ Department of Applied Mathematics \\ Nanjing University of Science and Technology \\ Nanjing 210094, People's Republic of China \\ Email: jinhuafan@hotmail.com
}

\begin{abstract}
In this paper, we prove that the asymptotic Teichmuller space of Riemann surfaces of analytically infinite type with the asymptotic Teichmuller metricis not Gromov hyperbolic.
\end{abstract}

Keywords-Riemannsurface, asymptotic Teichmuller space, Gromov hyperbolic, asymptotic extremal

\section{INTRODUCTION}

Let $\mathrm{R}$ be a hyperbolic Riemann surface, $\mathrm{T}(\mathrm{R})$ and $\mathrm{AT}(\mathrm{R})$ be the Teichmuller space and asymptotic Teichmuller space on $\mathrm{R}$. The study of asymptotic Teichmuller space was initiated by Gardiner and Sullivan in [6] for the case that $\mathrm{R}$ is the unit disk. General results on asymptotic Teichmuller space AT(R) were obtained in the papers [1] [2] [4] [17] and the book [5]. You can refer to the papers [2][14][15][16][17] for recently progress onasymptotic Teichmuller. Masur and Wolf [11] proved that the Teichmuller space of compact Riemann surfaces of genus $g>1$ with the Teichmuller metric is not Gromov hyperbolic. This result had been proved by other mathematicians in the papers [7] [12] [13]. The goal of this paper is to prove that asymptotic Teichmuller space with the asymptotic Teichmuller metric is not Gromov hyperbolic. For AT(R) has interesting only when $R$ is of analytically infinite type, otherwise, AT(R) consists of only one point. So, in what follows, we restrict $\mathrm{R}$ to be of analytically infinite type.

\section{PRELIMINARIES}

\section{A. Asymptotic Teichmuller Space}

The Teichmuller space $T(R)$ is the set of equivalence classes of quasiconformal mappings on R. Two mappings $f$ and $g$ are equivalent if there is a conformal mapping $\mathrm{c}$ from $\mathrm{f}(\mathrm{R})$ onto $\mathrm{g}(\mathrm{R})$ and a homotopy through quasiconformal mappings $h_{t}$ mappings $\mathrm{R}$ onto $\mathrm{g}(\mathrm{R})$ such that

$h_{0}=c \circ f, h_{1}=g$ and $h_{t}(p)=c ? f(p)=g(p)$

for every $\mathrm{p}$ in the ideal boundary of R.

The asymptotic Teichmuller space AT(R) has the same definition with one exception. The mapping $\mathrm{c}$ is allowed to be asymptotically conformal. A mapping $\mathrm{f}$ is asymptotically conformal if, for every $\varepsilon>0$, there is a compact subset $\mathrm{E}$ of $\mathrm{R}$ such that the dilatation of $\mathrm{f}$ outside of $\mathrm{E}$ is less than $1+\varepsilon$.
Let [f] or $[\mu]$ denote the equivalence class of the quasiconformal mapping class of the quasiconformal mapping $\mathrm{f}$ in the asymptotic Teichmuller space (we call this equivalence class be asymptotic equivalence class), where $\mu$ is the Beltrami differential of $\mathrm{f}$. Therefore, the asymptotic Teichmuller space AT(R) may be represented as the space of asymptotic equivalence classes of Beltrami differentials $\mu$ in the unit ball $\mathrm{M}(\mathrm{R})$ of the space $L^{\infty}(\mathrm{R})$.

Let $\mu \in M(R)$, we define $h^{*}(\mu)$ and $h([\mu])$ as $h^{*}(\mu)=\inf \left\{\left\|\left.v\right|_{R \backslash F}\right\|_{\infty}: F\right.$ is a compact subset of $\left.\mathrm{R}\right\}$, $h([\mu])=\inf \left\{\left\|\left.v\right|_{R \backslash F}\right\|_{\infty}: v \in[\mu], F\right.$ is a compact subset of $\mathrm{R}\}$.

We say $\mu$ is asymptotically extremal if $h^{*}(\mu)=h([\mu])$.

Given two points $[\mu]$ and $[v]$ in $\mathrm{AT}(\mathrm{R})$, the asymptotic Teichmuller metric $d([\mu],[v])$ between $[\mu]$ and $[v]$ is defined as

$$
d([\mu],[v])=\frac{1}{2} \log \frac{1+h\left(\left[f^{\mu}{ }_{\circ}\left(g^{v}\right)^{-1}\right]\right)}{1-h\left(\left[f^{\mu}{ }_{\circ}\left(g^{v}\right)^{-1}\right]\right)}
$$

\section{B. Degenerating sequence}

We denote by $A(R)$ the set of holomorphic quadratic differentials of $\mathrm{R}$ with norm

$$
\|\phi\|=\int_{R} \phi d x d y<\infty .
$$

By definition, a degenerating sequence in $A(R)$ is a sequence of quadratic differentials $\left\{\phi_{n}\right\}$ such that $\left\|\phi_{n}\right\|=1$ for all $\mathrm{n}$ and $\phi_{n} \rightarrow 0$ uniformly on compact subsets of $\mathrm{R}$ as $n \rightarrow \infty$. It is well known (see for instance Chapter 3 of [5]) that analytically infinite type of $R$ implies $A(R)$ contains degenerating sequences. It was proved in [2] that a Beltrami differential $\mu$ is asymptotically extremal if and only if there is a degenerating sequence $\left\{\phi_{n}\right\}$ such that

$$
h^{*}(\mu)=\lim _{n \rightarrow \infty}\left|\int_{R} \mu \phi_{n} d x d y\right| \text {. }
$$

There exists a special degenerating sequence $\left\{\phi_{n}\right\}$ which 
was constructed in the papers [8] [9] and [10] by Li Zhong and played an important role in papers [3] [8] [9] and [10]. The degenerating sequence is also important for our proof, we state it as

Theorem A. There exist a compact subset sequence $\left\{E_{n}\right\}$ of $R$ and a degenerating sequence $\left\{\phi_{n}\right\}$ which satisfy the following conditions (1)-(7).

$$
\begin{aligned}
& E_{n} \subset E_{n+1}, n=1,2, \ldots ; R=\cup_{n=1}^{\infty} E_{n} \\
& \left|\phi_{n}(z)\right|<2^{-n} \text { for all } \quad z \in E_{n-1}, \\
& \int_{E_{n} \backslash E_{n-1}\left|\phi_{n}\right|>1-2^{-n},} \\
& \int_{R \backslash E_{n}}\left|\phi_{m}\right|<2^{-n} \text { if } 1 \leq m \leq n, \\
& \int_{E_{n-1}}\left|\phi_{n}\right|<O\left(2^{-n}\right) \text { as } n \rightarrow \infty, \\
& \int_{E_{n} \backslash E_{n-1}}\left|\phi-\phi_{n}\right|<O\left(n 2^{-n}\right) \text { as } n \rightarrow \infty, \\
& \int_{E_{n} \backslash E_{n-1}}|\phi|<1+O\left(n 2^{-n}\right) \text { as } n \rightarrow \infty,
\end{aligned}
$$

where $\phi=\sum_{n=1}^{\infty} \phi_{n}$.

You can refer to the papers [8 ][9] and [10] for the proof of Theorem A.

\section{Gromov hyperbolicity}

The following definition of Gromov hyperbolicity comes from [10]. Let $\mathrm{X}$ be a geodesic metric space, that is, a metric space $\left(\mathrm{X}, d_{X}\right)$ where every pair of $A, B \in X$ can be connected by the isometric image of the segment $\left[0, d_{X}(A, B)\right]$. In such a space,we can define the notion of a triangle with vertices $\mathrm{A}, \mathrm{B}$ and $\mathrm{C}$ in $\mathrm{X}$ to be the union of geodesic segments $[\mathrm{AB}],[\mathrm{BC}]$ and $[\mathrm{AC}]$ which connecting $\mathrm{A}$ and $\mathrm{B}, \mathrm{B}$ and $\mathrm{C}$, and $\mathrm{A}$ and $\mathrm{C}$, respectively.

Definition 1. The geodesic metric space $X$ is Gromov hyperbolic if there is a number $\delta>0$ so that for every triangle $\Delta=[A B] \cup[B C] \cup[A C]$ and every $D \in[A B]$, we have $d_{X}(D,[B C] \cup[A C])<\delta$

By the result of Chapter 15 of [5], we know that there is at least one geodesic segment connecting any two given points, so the asymptotic Teichmuller space with the asymptotic Teichmuller metric is a metric space.

\section{MAIN THEOREM AND PROOF}

The goal of this section is to prove the following theorem.

Theorem1: Asymptotic Teichmuller space of Riemann surfaces of analytically infinite type with the asymptotic Teichmuller metric is not Gromov hyperbolic.

The main idea to prove Theorem 1 is to constructing a sequence of triangles so that the condition in Definition 1 dose not hold. To prove the theorem, we need the following lemmas.
Lemma 1. Let $\left\{E_{n}\right\},\left\{\phi_{n}\right\}$ and $\phi$ be constructed in Theorem A, and

$$
\mu(z)=\left\{\begin{array}{l}
k_{1} \frac{\overline{\phi(z)}}{|\phi(z)|}, z \in E_{2 n+1} \backslash E_{2 n}, \\
k_{2} \frac{\overline{\phi(z)}}{|\phi(z)|}, z \in E_{2 n} \backslash E_{2 n-1},
\end{array}\right.
$$

where $k_{1}, k_{2} \in(-1,1)$ are real constant. Then the Beltrami differential $\mu(z)$ is asymptotically extremal.

Proof. If $k_{1}>k_{2}$, then $h *(\mu)=k_{1}$, we claim that $\lim _{n \rightarrow \infty}\left|\int_{R} \mu \phi 2 n+1 d x d y\right|=h *(\mu)$, which implies the Lemma. For

$$
\begin{aligned}
\int_{R} \mu \phi 2 n+1= & \int_{R \backslash E_{2 n+1}} \mu \phi_{2 n+1}+\int_{E_{2 n+1} \backslash E_{2 n}} k_{1} \frac{\bar{\phi}}{|\phi|} \phi_{2 n+1} \\
& +\int_{E_{2 n}} \mu \phi_{2 n+1} .
\end{aligned}
$$

It following from (4) that

$$
\lim _{n \rightarrow \infty}\left|\int_{R \backslash E_{2 n+1}} \mu \phi_{2 n+1}\right| \leq\left|k_{1}\right| \lim _{n \rightarrow \infty} \int_{R \backslash E_{2 n+1}}\left|\phi_{2 n+1}\right|=0,
$$

so

$$
\lim _{n \rightarrow \infty} \int_{R \backslash E_{2 n+1}} \mu \phi_{2 n+1}=0 .
$$

It following from (5) that

$$
\lim _{n \rightarrow \infty}\left|\int_{E_{2 n+1}} \mu \phi_{2 n+1}\right| \leq\left|k_{1}\right| \lim _{n \rightarrow \infty} \int_{E_{2 n+1}}\left|\phi_{2 n+1}\right|=0,
$$

so

$$
\lim _{n \rightarrow \infty} \int_{E_{2 n}} \mu \phi_{2 n+1}=0 .
$$

It following from (6) and (7) that

$$
\begin{aligned}
& \int_{E_{2 n+1} \backslash E_{2 n}} k_{1} \frac{\bar{\phi}}{|\phi|} \phi_{2 n+1}=\int_{E_{2 n+1} \backslash E_{2 n}} k_{1} \frac{\bar{\phi}}{|\phi|} \phi \\
& +\int_{E_{2 n+1} \backslash E_{2 n}} k_{1} \frac{\bar{\phi}}{|\phi|}\left(\phi_{2 n+1}-\phi\right) \rightarrow k_{1}(n \rightarrow \infty)
\end{aligned}
$$

From (8)-(11), we conclude that

$$
\lim _{n \rightarrow \infty}\left|\int_{R} \mu \phi_{2 n+1}\right|=\left|k_{1}\right|=h^{*}(\mu)
$$

If $k_{2} \geq k_{1}$, by similar argument, we have

$$
\lim _{n \rightarrow \infty}\left|\int_{R} \mu \phi_{2 n}\right|=\left|k_{2}\right|=h *(\mu) \text {. }
$$

Which completes the proof.

Lemma 2. Let $f(t)=\frac{e^{2 t}-1}{e^{2 t}+1}, g(t)=\frac{t+k}{1+t k}$, where $k \in(-1,1)$ is a real constant. Then $g \circ f(t)$ is an isometry from $\left(\left[0, \log \frac{1+k}{1-k}\right], d_{E}\right)$ to $\left([-k, k], \rho_{\Delta}\right)$, where $d_{E}$ and $\rho_{\Delta}$ denote the Euclidean metric on $\left[0, \log \frac{1+k}{1-k}\right]$ and the Poincare metric $\rho_{\Delta}$ on unit disk respectively. 
Proof. For $g$ is a conformal mapping which preserve the unit disk, then $g$ is an isometry $\left(\left[0, \frac{2 k}{1+k^{2}}\right], \rho_{\Delta}\right)$ to $\left([-k, k], \rho_{\Delta}\right)$. By some computation, we know that $f(t)$ is an isometry from $\left(\left[0, \log \frac{1+k}{1-k}\right], d_{E}\right)$ to $\left(\left[0, \frac{2 k}{1+k^{2}}\right], \rho_{\Delta}\right)$, so $g \circ f(t)$ is isometric. Which completes the proof.

Let $\left\{E_{n}\right\}$ and $\phi$ be constructed in Theorem A, we define two Beltrami differentials on $R$ as

$$
v(z)=k \frac{\bar{\phi}}{|\phi|}
$$

and

$$
\eta(z)=\left\{\begin{array}{l}
k \frac{\overline{\phi(z)}}{|\phi(z)|}, z \in E_{2 n+1} \backslash E_{2 n}, n=0,1, \ldots \\
-k \frac{\overline{\phi(z)}}{|\phi(z)|}, z \in E_{2 n} \backslash E_{2 n-1}, n=1,2, \ldots
\end{array}\right.
$$

Denote $A=[v], B=[-v], c=[\eta]$ and $\alpha=\frac{1}{2} \log \frac{1+k}{1-k}$, from the definition of asymptotic Teichmuller metric and Lemma 1, we know that $d(A, B)=d(B, C)=d(A, C)=2 \alpha . \quad$ To prove Theorem 1, we will give an explicit formula for geodesic segments $[A B],[B C],[A C]$. By Lemma 2, we know that these geodesic segments are the isometric images of $\left([-k, k], \rho_{\Delta}\right)$.

Lemma 3. Let $\Gamma_{A B}(t)=\left[t \frac{\bar{\phi}}{|\phi|}\right]$, then $\Gamma_{A B}$ is an isometric image of $\left([-k, k], \rho_{\Delta}\right)$ which connects points $A$ and $B$.

Proof. It is easy to check that $\Gamma_{A B}(k)=A$ and $\Gamma_{A B}(-k)=B$. For any $t_{1}, t_{2} \in[-k, k]$,

$$
\frac{\Gamma_{A B}\left(t_{1}\right)-\Gamma_{A B}\left(t_{2}\right)}{1-\Gamma_{A B}\left(t_{1}\right) \overline{\Gamma_{A B}\left(t_{2}\right)}}=\frac{t_{1}-t_{2}}{1-t_{1} t_{2}} \frac{\bar{\phi}}{|\phi|} \text {. }
$$

I

By Lemma 1, we have

$$
d\left(\Gamma_{A B}\left(t_{1}\right), \Gamma_{A B}\left(t_{2}\right)\right)=\frac{1}{2} \log \frac{1+h\left(\left[\frac{t_{1}-t_{2}}{1-t_{1} t_{2}} \frac{\bar{\phi}}{|\phi|}\right]\right)}{1-h\left(\left[\frac{t_{1}-t_{2}}{1-t_{1} t_{2}} \frac{\bar{\phi}}{|\phi|}\right]\right)}
$$

$$
=\frac{1}{2} \log \frac{1+\left|\frac{t_{1}-t_{2}}{1-t_{1} t_{2}}\right|}{1-\left|\frac{t_{1}-t_{2}}{1-t_{1} t_{2}}\right|}=\rho_{\Delta}\left(t_{1}, t_{2}\right) .
$$

Which completes the proof.

\section{Lemma 4. Let}

$$
\Gamma_{A C}(t)=\left\{\begin{array}{l}
k \frac{\overline{\phi(z)}}{|\phi(z)|}, z \in E_{2 n+1} \backslash E_{2 n}, n=0,1, \ldots \\
-t \frac{\overline{\phi(z)}}{|\phi(z)|}, z \in E_{2 n} \backslash E_{2 n-1}, n=1,2, \ldots
\end{array}\right.
$$

then $\Gamma_{A C}$ is an isometric image of $\left([-k, k], \rho_{\Delta}\right)$ which connects points $A$ and $C$.

Proof. It is easy to check that $\Gamma_{A C}(k)=A$ and $\Gamma_{A C}(-k)=C$. For any $t_{1}, t_{2} \in[-k, k]$,

$\frac{\Gamma_{A C}\left(t_{1}\right)-\Gamma_{A C}\left(t_{2}\right)}{1-\Gamma_{A C}\left(t_{1}\right) \overline{\Gamma_{A C}\left(t_{2}\right)}}=\left\{\begin{array}{l}0, z \in E_{2 n+1} \backslash E_{2 n}, n=0,1, \ldots \\ \frac{t_{1}-t_{2}}{1-t_{1} t_{2}} \frac{\overline{\phi(z)}}{|\phi(z)|}, z \in E_{2 n} \backslash E_{2 n-1}, n=1,2, \ldots\end{array}\right.$

By Lemma 1, we have

$$
d\left(\Gamma_{A C}\left(t_{1}\right), \Gamma_{A C}\left(t_{2}\right)\right)=\frac{1}{2} \log \frac{1+\left|\frac{t_{1}-t_{2}}{1-t_{1} t_{2}}\right|}{1-\left|\frac{t_{1}-t_{2}}{1-t_{1} t_{2}}\right|}=\rho_{\Delta}\left(t_{1}, t_{2}\right) .
$$

Which completes the proof.

By the same argument, we have the similar following lemma.

Lemma 5. Let

$$
\Gamma_{B C}(t)=\left\{\begin{array}{l}
t \frac{\overline{\phi(z)}}{|\phi(z)|}, z \in E_{2 n+1} \backslash E_{2 n}, n=0,1, \ldots \\
-k \frac{\overline{\phi(z)}}{|\phi(z)|}, z \in E_{2 n} \backslash E_{2 n-1}, n=1,2, \ldots
\end{array},\right.
$$

then $\Gamma_{B C}$ is an isometric image of $\left([-k, k], \rho_{\Delta}\right)$ which connects points $B$ and $C$.

From Lemma3-Lemma5, we know that $\Gamma_{A B}(t)$, $\Gamma_{B C}(t)$ and $\Gamma_{A C}(t)$ can be regarded as an explicit formula for the geodesic segment $[\mathrm{AB}],[\mathrm{BC}]$ and $[\mathrm{AC}]$ respectively.

\section{Proof of Theorem 1.}

Let $\Delta=[A B] \cup[B C] \cup[A C]$ be a triangle in the asymptotic Teichmuller space AT(R), where $[\mathrm{AB}],[\mathrm{AC}]$ and $[\mathrm{BC}]$ be the same as them in Lemma 3-Lemma 5 respectively. Let $D=[0]=\Gamma_{A B}(0) \in[A B]$, by Lemma 4,5 and Lemma 1 , then 


$$
d(D,[B C] \cup[A C])=\frac{1}{2} \log \frac{1+k}{1-k} .
$$

As $k \rightarrow 1$, we see $d(D,[B C] \cup[A C]) \rightarrow \infty$. Which completes the proof.

From the proof of Theorem 1, the following corollary is obvious.

Corollary 1. Any ball in an asymptotic Teichmuller space of Riemann surfaces of analytically infinite type is not strictly convex with respect to geodesics.

\section{Proof.}

For the Ball $B([0], r)=\{p \in A T(R), d([0], p) \leq r\}$, where $r=\frac{1}{2} \log \frac{1+k}{1-k}, \Gamma_{A C}(t)$ and $\Gamma_{B C}(t)$ in Lemma 4 and 5 are geodesics on the

$$
S([0], r)=\{p \in A T(R), d([0], p)=r\},
$$

which implies the result of the corollary.

\section{REFERENCES}

[1] C. J. Earle, F. P. Gardiner, and N. Lakic, "Asymptotic Teichmuller space. I. The complex Structure," In the tradition of Ahlfors and Bers, Contemp. Math., 256, Amer. Math. Soc., Providence, RI, 2000, pp. 17-38.

[2] C. J. Earle, F. P. Gardiner and N. Lakic, "Asymptotic Teichmuller space. II. The metric structure," In the tradition of Ahlfors and Bers III, Contemp. Math. 355, Amer. Math. Soc., Providence, RI, 2004. pp. 187-219.

[3] C. J. Earle and Z. Li, “ Isometrically embedded polydisks in infinite dimensional Teichmuller spaces, ” J. Geom. Anal. Vol. 9, pp. 51-71, 1999.

[4] C. J. Earle, V. Markovic and D, Saric, “ Barycentric extension and the Bers embedding for asymptotic Teichmuller space, " In Complex manifolds and hyperbolic geometry, Contemp. Math. 311, Amer. Math. Soc., Providence, RI, pp. 87-105, 2002.

[4] F. P. Gardiner and N. Lakic, Quasiconformal Teichmuller theory, Mathematical Surveys and Monographs 76. American Mathematical Society, Providence, RI, 2000.

[5] F. P. Gardiner and D. Sullivan, Symmetric structures on a closed curve, Amer. J. Math. vol.114, pp.683-736, 1992.

[6] N. V. Ivanov, A short proof of non-Gromov hyperbolicity of Teichmuller spaces, Ann. Acad. Sci. Fenn. Ser. A I Math. vol. 27, pp.3-5, 2002.

[6] Z. Li, Closed geodesics and non-differentiability of the metric in infinite-dimensionalTeichmuller spaces, Proc. Amer. Math. Soc. vol.124, 1459-1465, 1996.

[7] Z. Li, Non-convexity of spheres in infinite-dimensional Teichmuller spaces, Sci. China Ser. A vol. 37, pp.924-932, 1994.

[8] Z. Li, A note on geodesics in infinite-dimensional Teichmuller, Ann. Acad. Sci. Fenn. Ser. A I Math. vol. 20, pp.301-313, 1995.

[9] H. A. Masur and M. Wolf, Teichmuller space is not Gromov hyperbolic, Ann. Acad. Sci. Fenn. Ser. A I Math. vol. 20, pp.359-267, 1995.

[10] J. D. McCarthy and A. Papadopoulos, The visual sphere of Teichmuller space and a theorem of Masur Wolf, Ann. Acad. Sci. Fenn. Ser. A I Math.vol. 24 , pp. 147-154, 1999.

[11] J. D. McCarthy and A. Papadopoulos, The mapping class group and a theorem of Masur-Wolf, Topology Appl. Vol. 96, 75-84,1999.

[12] H. Miyachi, On invariant distances on asymptotic Teichmuller spaces, Proc. Amer. Math. Soc. Vol.134, 1917-1925,2006.

[13] H. Miyachi, Image of asymptotic Bers Map, J. Math.Soc. Japan, vol. $60,1255-1276,2008$.

[14] H. Miyachi, The inner and outer radii of asymptotic Teichmuller spaces, Complex Variables and Elliptic Equations. vol.53, 139-158, 2008.

[15] G. Yao, Harmonic maps and asymptotic Teichmuller space, Manuscripta Math. vol. 122, 375-389, 2007. 